Relationship of nutritional status, inflammation, and serum albumin levels during acute illness: A prospective study

Andreas Eckart M.D. , Tristan Struja M.D. , Alexander Kutz M.D. , Annic Baumgartner M.D. , Thomas Baumgartner ,

Seline Zurfluh M.D. , Olivia Neeser, Andreas Huber M.D. Prof , Zeno Stanga M.D. Prof, Beat Mueller M.D. Prof , Philipp Schuetz M.D., MPH Prof

PII: S0002-9343(19)30975-1

DOI: https://doi.org/10.1016/j.amjmed.2019.10.031

Reference: AJM 15475

To appear in:

The American Journal of Medicine

Please cite this article as: Andreas Eckart M.D., Tristan Struja M.D. , Alexander Kutz M.D. , Annic Baumgartner M.D. , Thomas Baumgartner , Seline Zurfluh M.D. , Olivia Neeser, Andreas Huber M.D. Prof , Zeno Stanga M.D. Prof, $\quad$ Beat Mueller M.D. Prof, Philipp Schuetz M.D., MPH Prof, Relationship of nutritional status, inflammation, and serum albumin levels during acute illness: A prospective study, The American Journal of Medicine (2019), doi: https://doi.org/10.1016/j.amjmed.2019.10.031

This is a PDF file of an article that has undergone enhancements after acceptance, such as the addition of a cover page and metadata, and formatting for readability, but it is not yet the definitive version of record. This version will undergo additional copyediting, typesetting and review before it is published in its final form, but we are providing this version to give early visibility of the article. Please note that, during the production process, errors may be discovered which could affect the content, and all legal disclaimers that apply to the journal pertain.

(C) 2019 Published by Elsevier Inc. 


\section{Relationship of nutritional status, inflammation, and serum albumin levels during acute illness: A prospective study}

Andreas Eckart, M.D. ${ }^{\mathrm{a}, *}$; Tristan Struja, M.D. ${ }^{\mathrm{a}, \mathrm{d}}$; Alexander Kutz, M.D. ${ }^{\mathrm{a}}$; Annic Baumgartner, M.D. ${ }^{a, d}$; Thomas Baumgartner ${ }^{\mathrm{a}, 1}$; Seline Zurfluh, M.D. ${ }^{\mathrm{a}}$; Olivia Neeser ${ }^{\mathrm{a}}$; Andreas Huber, Prof,

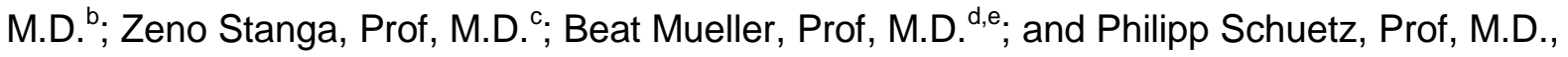
$\mathrm{MPH}^{\mathrm{a}, \mathrm{e}}$

eckartan@gmail.com; tristan.struja@ksa.ch; kutz.alexander@gmail.com;

annic.baumgartner@ksa.ch; thomas.baumgartner@insel.ch; seline.zurfluh@ksa.ch;

olivia.neeser@ksa.ch; andreas.huber@ksa.ch; zeno.stanga@insel.ch;

happy.mueller@unibas.ch; schuetzph@gmail.com

Author Affiliations: ${ }^{a}$ Division of General Internal and Emergency Medicine; University Department of Medicine, Kantonsspital Aarau, Tellstrasse 25, 5001 Aarau, Switzerland; ${ }^{b}$ Department of Laboratory Medicine, Kantonsspital Aarau, Tellstrasse 25, 5001 Aarau, Switzerland; 'Department of Diabetes, Endocrinology, Clinical Nutrition and Metabolism, Inselspital, Bern University Hospital, and University of Bern, Freiburgstrasse, 3010 Bern, Switzerland; 'Division of Endocrinology, Diabetes and Metabolism; University Department of Medicine, Kantonsspital Aarau, Tellstrasse 25, 5001 Aarau, Switzerland; ${ }^{\text {Medical Faculty, }}$ University of Basel, Basel, Switzerland;

${ }^{1}$ Present address: Department of Cardiology, Bern University Hospital, Inselspital, Bern, Switzerland

\section{${ }^{*}$ Corresponding author:}

Postal address: Division of General Internal and Emergency Medicine, University Department of Medicine, Kantonsspital Aarau, Tellstrasse 25, 5001 Aarau, Switzerland Fax: +41 6283898 73; Phone: +41 6283895 17; E-mail: eckartan@gmail.com 
Running headline: Relationship of nutritional status, inflammation, albumin

Keywords: Nutritional risk, inflammation, serum albumin, mortality

Word count: Abstract: 247; Main: 2,715

Number of Tables: 3; Number of Figures: 2; Number of References: 34

Number of Supplemental Tables: 1; Number of Supplemental Figures: 2 


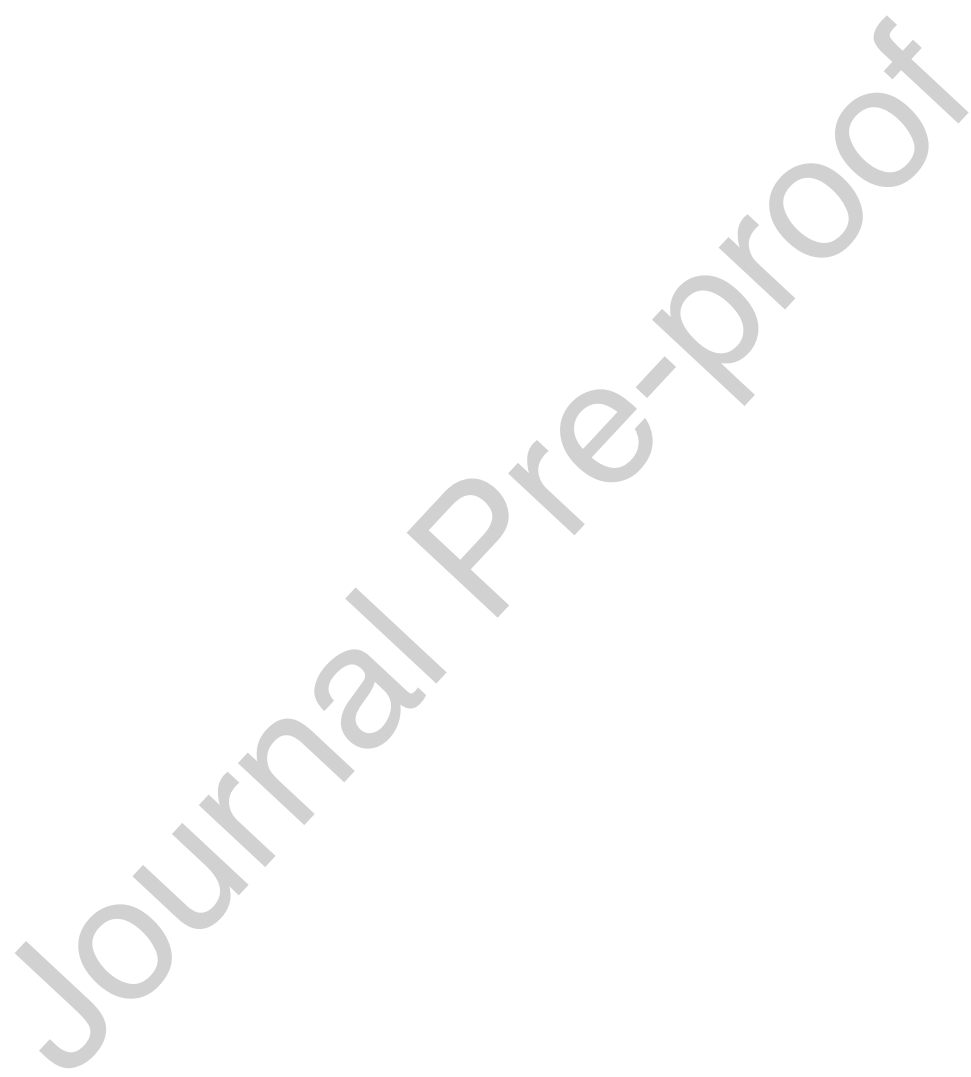




\section{Clinical Significance}

- Both inflammation and elevated nutritional risk (as assessed by the Nutritional Risk Screening 2002) were independently associated with hypoalbuminemia in acutely ill patients.

- At time of admission all of the three parameters namely inflammation, elevated nutritional risk, and hypoalbuminemia were independently associated with all-cause 30-day mortality.

- Combining these three parameters for the initial evaluation of a patient improved risk stratification. 


\section{Abstract \\ Background}

Low serum albumin levels resulting from inflammation-induced capillary leakage or diseaserelated anorexia during acute illness are associated with poor outcomes. We investigated the relationship of nutritional status and inflammation with low serum albumin levels and 30-day mortality in a large cohort.

\section{Methods}

We prospectively enrolled adult patients in the medical emergency department of a Swiss tertiary care center and investigated associations of C-reactive protein (CRP) and Nutritional Risk Screening (NRS 2002) as markers of inflammation and poor nutritional status, respectively, with low serum albumin levels and mortality using multivariate regression analyses.

\section{Results}

Of 2,465 patients, 1,019 (41\%) had low serum albumin levels (<34 g/L), $619(25.1 \%)$ had increased nutritional risk (NRS $2002 \geq 3$ ), and 1,086 (44.1\%) had CRP values $>20 \mathrm{mg} / \mathrm{L}$. Multivariate analyses adjusted for age, gender, diagnosis, and comorbidities revealed elevated CRP values (adjusted odds ratio [OR] 10.51, 95\% confidence intervals [CI] 7.51 to 14.72, $P<0.001$ ) and increased malnutrition risk (adjusted OR 2.87, 95\% $\mathrm{Cl} 1.98$ to 4.15 , $P<0.001$ ) to be associated with low serum albumin levels, even adjusting for both parameters. Low serum albumin levels, elevated CRP values, and increased nutritional risk independently predicted 30-day mortality, with areas under the curve (AUCs) of $0.77,0.70$, and 0.75 , respectively. Combination of these three parameters showed an AUC of 0.82 to predict mortality.

\section{Conclusions}

Elevated parameters of inflammation and high nutritional risk were independently associated with hypoalbuminemia. All three parameters independently predicted mortality. Combining them during initial evaluation of patients in emergency departments facilitates mortality risk stratification. 


\section{Introduction}

Hypoalbuminemia is common in hospitalized patients and is associated with adverse clinical outcomes. Increased mortality was documented in patients with low serum albumin levels in both hospital ${ }^{1-3}$ and community settings ${ }^{4}$. Serum albumin has been broadly used as a diagnostic marker for malnutrition in clinical practice since 1979 when Seltzer et al first proposed serum albumin as one of two components-the other being total lymphocyte count-forming the basis of an instant nutritional assessment ${ }^{5}$. Low serum albumin levels were initially thought to result from protein malnutrition or "kwashiorkor-like" malnutrition ${ }^{6}$. However, in the last 20 years, the relevance of serum albumin as a specific nutritional marker has been questioned ${ }^{7-9}$. Many different factors affect serum albumin levels, and it has been shown to lack sensitivity and specificity as an indicator of nutritional status ${ }^{8,10}$. Specifically, acute and chronic inflammatory conditions affect serum albumin levels by altering hepatic protein metabolism and inducing capillary leakage ${ }^{10-12}$. This being the case, serum albumin is not anymore considered a reliable nutritional marker in inflammatory states but rather a marker for disease severity ${ }^{13,14}$.

The role of albumin in non-inflammatory states is also in dispute since it is possible to die of starvation with normal serum albumin levels ${ }^{9,14}$.

In view of the above findings, the international Societies of Clinical Nutrition and Metabolism (European Society of Clinical Nutrition and Metabolism [ESPEN] and American Society of Clinical Nutrition and Metabolism [ASPEN]) no longer recommend assessment of serum albumin or any other biomarkers to identify and document adult malnutrition, choosing instead to focus on "insufficient energy intake", weight loss, anthropometric as well as functional measures ${ }^{13}$. In the absence of a broad accepted definition of malnutrition several nutritional screening tools as the validated Nutritional Risk Screening (NRS 2002) have been developed ${ }^{15}$. 
Despite these facts, many clinicians still believe that hypoalbuminemia is indicative of impaired nutritional status. Not surprisingly, serum albumin continues to be used as a marker of nutritional status, even in recent investigations ${ }^{16-18}$. In an attempt to shed more light on this topic, we investigated whether inflammation or poor nutritional status affect serum albumin levels in a large cohort of unselected adult patients seeking emergency medical care. Furthermore, we investigated whether inflammation, nutritional risk, and low serum albumin levels were independently associated with mortality. 


\section{Materials and Methods}

Study design and study population

This report presents the findings of secondary analysis of data from a prospective, observational, multicenter, multinational trial (the "TRIAGE" Study) that aimed to optimize triage and anticipate the post-acute care needs of adult patients seeking emergency medical care ${ }^{19}$. The TRIAGE trial included consecutive patients seen in the emergency department (ED) of three tertiary care hospitals in Paris (France), Switzerland (Aarau), and the USA (Clearwater, Florida) between March 2013 and February 2014. The Institutional Review Boards of the three centers approved the study and waived the need for informed consent since this secondary analysis was an observational quality control study. The main study was registered at ClinicalTrials.gov (NCT01768494).

This analysis included only adult inpatients of the Swiss center (Kantonsspital Aarau) seeking ED care for an acute medical illness. Of 3.586 patients, 1,121 were excluded due to missing data for either serum albumin, CRP, or parameters of the NRS 2002. 2,465 patients were included in the final analysis.

\section{Assessment of nutritional status and definition of nutritional impairment}

Nutritional status was assessed within 48 hours of hospital admission using the validated NRS 2002 tool in a bedside patient interview as recommended ${ }^{20}$. Patients with an NRS 2002 score of $\geq 3$ points were considered at risk for malnutrition, as proposed in the original publication ${ }^{15}$.

\section{Research objective and outcome measures}

The objective of this analysis was to investigate the interaction of nutritional status and inflammation with serum albumin levels. Therefore, the primary endpoint was the association of nutritional status (assessed by the NRS 2002) and inflammation (represented by CRP values) with serum albumin levels. Secondary endpoints were associations of inflammation 
(represented by CRP values), nutritional risk (represented by NRS 2002 total scores), and low serum albumin levels, respectively, with all-cause 30-day mortality.

\section{Statistical analysis}

Patient characteristics were expressed using descriptive statistics including mean with standard deviation (SD), median with interquartile range (IQR), and frequencies, as appropriate. Frequency comparison was done using the $\chi^{2}$ test, while correlations were assessed by Pearson rank correlation coefficients.

We used multivariate logistic regression with stepwise adjustment for important confounders (age, sex, comorbidities, and main medical diagnosis) to investigate associations with outcomes. We used receiver operating statistics, reporting area under the curve (AUC) as a measure of discrimination. We repeated analyses in predefined subgroups.

All tests were two-tailed and carried out at $5 \%$ significance levels. Analyses were performed with STATA 12.1 (Stata Corp., College Station, TX, USA).

Detailed information regarding Materials and Methods are provided in the supplementary material. 


\section{Results}

Study population

Overall, 2,465 patients with complete admission values for serum albumin, CRP, and NRS 2002 parameters were included in the final analysis. The median age was 71 years and $43.7 \%$ of patients were female. The median serum albumin concentration was $35.2 \mathrm{~g} / \mathrm{L}$. Overall, 1,019 (41\%) patients had low serum albumin levels (<34 g/L), $619(25.1 \%)$ were nutritionally at risk with NRS 2002 scores of 3 points $(333,13.5 \%)$ or higher $(286,11.6 \%)$, while 589 (23.9\%) patients had low to moderately elevated CRP values (5.01 to $20 \mathrm{mg} / \mathrm{L})$ and 1,086 (44.1\%) patients had markedly elevated CRP values (>20mg/L).

Compared to patients with normal serum albumin levels, patients with low serum albumin levels were older (72 y vs 70 y, $P<0.001)$, were more often at nutritional risk $(37.1 \%$ vs $16.7 \%, P<0.001$ ), and had significantly higher CRP values (median values of $67 \mathrm{mg} / \mathrm{L}$ vs 6 $\mathrm{mg} / \mathrm{L}, P<0.001)$.

Additional baseline characteristics of the overall population, stratified by admission serum albumin values, and by survival status are shown in Table 1.

Interaction of inflammation, nutritional risk, and low serum albumin

We found a significant negative correlation of serum albumin with CRP (Spearman's $\rho-0.62$, $P<0.001)$. Furthermore, we found a significant but weaker negative correlation of serum albumin with NRS 2002 total scores (Spearman's $\rho-0.33, P<0.001$ ) and a positive correlation between higher CRP values and higher NRS 2002 total scores (Spearman's $\rho$ $0.24, P<0.001)$ (Fig. 1).

Univariate regression analyses revealed that the frequency of hypoalbuminemia was more than 2.5 times higher in patients with mild to moderate elevated CRP values (5.01 to 20 $\mathrm{mg} / \mathrm{L}$ ) compared to those with normal CRP values (32.4\% vs $12.7 \%$ ), corresponding to an 
OR of $3.31(95 \% \mathrm{Cl} 2.52$ to $4.34, P<0.001)$ and more than 5 times higher in patients with markedly elevated CRP values (>20 mg/L) compared to those with normal CRP values (67.0\% vs $12.7 \%)$, corresponding to an OR of $14.03(95 \% \mathrm{Cl} 10.98$ to $17.93, P<0.001)$.

When associations of high nutritional risk and low serum albumin levels were examined, we found that occurrence of hypoalbuminemia in patients with an NRS 2002 total score of 3 points was increased by $50 \%$ compared to those with a score $<3$ points ( $53.8 \%$ vs $34.7 \%)$, corresponding to a univariate OR of $2.19(95 \% \mathrm{Cl} 1.73$ to $2.77, P<0.001)$. The frequency of hypoalbuminemia doubled in patients with an NRS 2002 total score $>3$ points compared to those with a score $<3(69.6 \%$ vs $34.7 \%)$, corresponding to a univariate OR of $4.30(95 \% \mathrm{Cl}$ 3.28 to $5.63, P<0.001)$.

After stepwise adjustment for important confounders (age, gender, comorbidities, and main diagnosis), both elevated CRP values and elevated nutritional risk remained significantly associated with depressed serum albumin levels, with fully adjusted ORs of $9.96(95 \% \mathrm{Cl}$ 7.10 to $13.98, P<0.001)$ and $2.40(95 \% \mathrm{Cl} 1.62$ to $3.54, P<0.001)$, respectively (Table 2$)$.

The ROC statistics for the prediction of hypoalbuminemia showed a higher predictive value for CRP values (AUC of $0.81 ; 95 \%$ Cl 0.79 to 0.82 ) than for NRS 2002 total scores (AUC of $0.65 ; 95 \% \mathrm{Cl} 0.62$ to 0.67 ). Combination of both parameters showed minimal to no improvement for the prediction of depressed serum albumin levels (AUC 0.82; $95 \% \mathrm{CI} 0.80$ to 0.84 ) (Table 2, Fig. 2A).

Associations of inflammation, nutritional risk, and low serum albumin with mortality Of the included 2,465 patients, 205 (8.3\%) had died by follow up 30 days after admission. Mortality was 4.5 times higher in patients with hypoalbuminemia compared to those with normal serum albumin levels (15.4\% vs 3.3\%), 5 times higher in patients with CRP values $>20 \mathrm{mg} / \mathrm{L}$ compared to patients with normal CRP values (<5 mg/L) $(13.4 \%$ vs $2.5 \%)$, and 
more than 6 times higher in patients with NRS 2002 total scores $>3$ points compared to those with NRS 2002 scores $<3$ points $(26.6 \%$ vs $4.2 \%)$.

In adjusted regression analyses, all three parameters (i.e., hypoalbuminemia, CRP values, and NRS 2002 scores) were significantly and independently associated with mortality, with corresponding fully adjusted ORs of $2.87(95 \% \mathrm{Cl} 1.70$ to $4.84, P<0.001)$ for hypoalbuminemia (<34 g/L vs normal), $3.23(95 \% \mathrm{Cl} 1.52$ to $6.87, P=0.002)$ for $\mathrm{CRP}$ (>20mg/L vs normal), and $4.56(95 \% \mathrm{Cl} 2.75$ to $7.58, P<0.001)$ for NRS 2002 total scores (>3 points vs $<3$ points) (Table 3 ).

The ROC statistics showed low serum albumin levels, increased CRP values, and higher NRS 2002 total scores to be independent predictors of 30-day mortality, with AUCs of 0.77 (95\% $\mathrm{Cl} 0.74$ to 0.81$), 0.70$ (95\% $\mathrm{Cl} 0.66$ to 0.73$)$, and 0.75 (95\% Cl 0.72 to 0.79 ), respectively. A combined model including these three parameters revealed an AUC of 0.82 (95\% $\mathrm{Cl} 0.79$ to 0.84 ) to predict mortality (Table 3, Fig. $2 \mathrm{~B}$ ).

\section{Subgroup analyses}

Subgroup analyses are shown in the appendix (Figs. A1 and A2). Associations of nutritional risk and inflammation, respectively, with hypoalbuminemia were similar irrespective of CRP values, NRS 2002 total scores, and main diagnoses leading to hospitalization (Figs. A1A and A1B).

We also found no difference with regard to associations of hypoalbuminemia, elevated nutritional risk, and different states of inflammation, respectively, with mortality in subgroups stratified by CRP values, NRS 2002 scores, serum albumin levels, and main diagnoses leading to hospitalization (Figs. A2A to A2C). 


\section{Discussion}

The main findings of this study are threefold. First, we found low serum albumin levels, elevated nutritional risk, and elevated CRP values to be frequently seen in a large cohort of unselected medical inpatients at time of admission. Second, we found both inflammation and elevated nutritional risk to be independently associated with hypoalbuminemia. Finally, we found low serum albumin levels, elevated CRP values, and nutritional risk to be independent predictors of mortality.

Prevalence of hypoalbuminemia at time of admission in our cohort $(41 \%)$ was similar to that reported in an Italian study (46.5\%) investigating prevalence of hypoalbuminemia in an internal medicine department ${ }^{23}$, but was higher than the $29 \%$ found in a large Israeli study also investigating patients admitted to the medical ward ${ }^{1}$. This discrepancy could be due to differences in healthcare systems. In our study, mortality in patients with hypoalbuminemia at admission (15.4\%) was also consistent with findings of other investigations that reported mortality rates between $14.0 \%$ and $16.3 \%$ among hospitalized patients ${ }^{1-3}$.

Whether serum albumin levels in sick patients are influenced mainly by inflammation or by malnutrition has long been discussed. Most of the existing evidence suggest that serum albumin is not an appropriate parameter to determine the nutritional status ${ }^{24}$ and that changes in acute phase proteins such as serum albumin and prealbumin do not reflect poor nutritional status but rather the severity of inflammation and illness in acute disease ${ }^{8,25-27}$. In our study, we confirm that inflammation is associated with hypoalbuminemia. This is explained by underlying processes in inflammation, wherein serum albumin levels decrease due to several mechanisms ${ }^{8,27,28}$.

Further, we found an association of nutritional risk with low serum albumin values, independent of the inflammatory status. This is in line with findings of a few recent investigations in which serum albumin is still considered a suitable marker to identify 
malnutrition in patients after heart transplantation ${ }^{17}$, and before orthopedic surgery ${ }^{16}$. Another article notes the value of serum albumin as a nutritional screening marker in maintenance dialysis patients ${ }^{18}$. Authors emphasize low cost and easy availability of serum albumin as a screening marker as compared to other nutritional assessment which generally require extensive training and clinical skills.

In contrast, investigations in healthy individuals suggest that in states of starvation, serum albumin has no value as a marker to identify malnourished patients and their need for feeding. In a recent systematic review, serum albumin levels failed to identify subjects with severe protein-energy malnutrition until states of obvious starvation (e.g., BMI below 12 $\mathrm{kg} / \mathrm{m}^{2}$ or prolonged fasting) had been reached ${ }^{9}$. This is in line with a study of The Academy of Nutrition and Dietetics Evidence Analysis Library (EAL) that did not find a consistent change in serum albumin levels with weight loss or caloric restriction ${ }^{29}$. However, our results indicate that at least in individuals experiencing acute diseases, elevated nutritional risk seems to have an impact on serum albumin levels, independent of the inflammatory state.

Our results further indicate that hypoalbuminemia, elevated nutritional risk, and elevated CRP values are independent predictors of mortality. Interestingly, combining the three parameters namely serum albumin levels, CRP values, and NRS 2002 total scores for the initial evaluation of patients even improved risk stratification.

Our finding of heightened nutritional risk (evaluated using the NRS 2002 tool) as a mortality predictor is in line with findings of a large Polish study in which the in-hospital mortality rate in patients with NRS 2002 total scores $\geq 3$ points (19.7\%) was similar to the 30 -day mortality rate $(20.7 \%)$ in our patients with elevated risk of malnutrition ${ }^{30}$.

As for the association of serum albumin levels with mortality, our findings are in line with the results of a meta-analysis ${ }^{31}$ indicating an association between hypoalbuminemia and 
mortality that was independent of both nutritional status and inflammation. However, there was no standardized nutritional assessment in the studies included in the meta-analysis, and different nutritional indices such as BMI, body weight, body fat percentage, and midarm circumference had been used. To the best of our knowledge, the present study is the first to show an association of serum albumin levels with mortality independent of both inflammation and nutritional risk as assessed by NRS 2002.

Our study has a few limitations. First, as a secondary analysis of data from a previous trial, the results are primarily hypothesis generating. Confounding between the crude associations of serum albumin levels, CRP values, and nutritional risk is likely, although we did address this limitation by adjusting for important confounders. Second, due to exclusion of about one third of the patients there is the possibility of a selection bias. However, patient characteristics of the excluded patients were comparable to those included (Table A1). Third, we assessed laboratory parameters and nutritional risk only at the time of admission and did not record nutritional interventions such as use of nutritional (oral) supplements. Thus, we were not able to demonstrate whether improvement of nutritional status or changes in laboratory parameters translate into better clinical outcomes. Fourth, besides the NRS 2002, we did not perform an additional detailed assessment of patients' nutritional status. Not all patients with increased nutritional risk may have clinically overt malnutrition and may have been missed by the NRS 2002 tool. However, it is an extensively used evidence-based screening tool that has been thoroughly validated ${ }^{21,22}$ and is recommended by the ESPEN as a suitable tool for screening adult inpatients ${ }^{8,20}$. The NRS 2002 was found to have a positive predictive value of 79 to $85 \%$ for the prediction of undernutrition in hospitalized patients ${ }^{21,32,33}$. Finally, acute inflammatory disease contributes to higher NRS 2002 total scores, as one part of the score is influenced by the severity of illness (stress metabolism). Thus, there is the possibility of some kind of interaction between NRS 2002 total scores and inflammation. 


\section{Conclusions}

This prospective study that included medical patients upon ED admission found elevated parameters of inflammation as well as nutritional risk to be independently associated with hypoalbuminemia. This suggests that both inflammation and nutritional risk contribute to low serum albumin levels in acutely ill patients. However, the impact of inflammation seems to be stronger. All three parameters namely hypoalbuminemia, high CRP values, and NRS 2002 total scores $\geq 3$ were independent predictors of mortality. Combining these parameters for the initial evaluation of a patient thus facilitates improved risk stratification.

\section{Author's Contributions}

AE and PS had complete access to all study data and take full responsibility for the integrity of the data and the accuracy of the analyses. All authors made substantive intellectual contributions to this study regarding conception and design of the study, and were responsible for the acquisition, analysis, and interpretation of the data. AE, TS and PS performed the statistical analyses and drafted the first manuscript. All authors approved the final version of the manuscript and the decision to submit the manuscript for publication.

Declaration of interest: $A K, B M$, and PS received support from B.R.A.H.M.S AG (now ThermoFisher Scientific Biomarkers) and bioMérieux for meetings, speaking engagements, and research grants. BM has served as a consultant to both companies.

Funding/Support: ThermoFisher provided an unrestricted research grant for the TRIAGE study. PS is supported by the Swiss National Science Foundation (SNSF Professorship, PP00P3_150531/1) and the Research Council of the Kantonsspital Aarau (1410.000.044).

Role of the Funder/Sponsor: The funders/sponsors had no role in the design or conduct of the study; preparation, review, or approval of the manuscript; or decision to submit the manuscript for publication. 
Acknowledgements: We thank the patients whose data are presented here for their participation in the main "TRIAGE" study. Editorial assistance during the preparation of the manuscript was provided by Prasad Kulkarni, PhD, CMPP of Asclepius Medical Communications LLC, Ridgewood, NJ, USA and was funded by the authors. 


\section{References}

1. Akirov A, Masri-Iraqi H, Atamna A, Shimon I. Low Albumin Levels Are Associated with Mortality Risk in Hospitalized Patients. The American journal of medicine. 2017;130(12):1465.e1411-1465.e1419.

2. Herrmann FR, Safran C, Levkoff SE, Minaker KL. Serum albumin level on admission as a predictor of death, length of stay, and readmission. Archives of internal medicine. 1992;152(1):125-130.

3. Jellinge ME, Henriksen DP, Hallas P, Brabrand M. Hypoalbuminemia is a strong predictor of 30-day all-cause mortality in acutely admitted medical patients: a prospective, observational, cohort study. PloS one. 2014;9(8):e105983.

4. Ferguson RP, O'Connor P, Crabtree B, Batchelor A, Mitchell J, Coppola D. Serum albumin and prealbumin as predictors of clinical outcomes of hospitalized elderly nursing home residents. Journal of the American Geriatrics Society. 1993;41(5):545549.

5. Seltzer MH, Bastidas JA, Cooper DM, Engler P, Slocum B, Fletcher HS. Instant nutritional assessment. JPEN Journal of parenteral and enteral nutrition. 1979;3(3):157-159.

6. Golden $\mathrm{MH}$. Evolution of nutritional management of acute malnutrition. Indian pediatrics. 2010;47(8):667-678.

7. Fuhrman MP. The albumin-nutrition connection: separating myth from fact. Nutrition (Burbank, Los Angeles County, Calif). 2002;18(2):199-200.

8. Fuhrman MP, Charney P, Mueller CM. Hepatic proteins and nutrition assessment. Journal of the American Dietetic Association. 2004;104(8):1258-1264.

9. Lee JL, Oh ES, Lee RW, Finucane TE. Serum Albumin and Prealbumin in Calorically Restricted, Nondiseased Individuals: A Systematic Review. The American journal of medicine. 2015;128(9):1023.e1021-1022.

10. Levitt DG, Levitt MD. Human serum albumin homeostasis: a new look at the roles of synthesis, catabolism, renal and gastrointestinal excretion, and the clinical value of serum albumin measurements. International journal of general medicine. 2016;9:229255.

11. Fleck A, Raines G, Hawker F, et al. Increased vascular permeability: a major cause of hypoalbuminaemia in disease and injury. Lancet (London, England). 1985;1(8432):781-784.

12. Franch-Arcas $G$. The meaning of hypoalbuminaemia in clinical practice. Clinical nutrition (Edinburgh, Scotland). 2001;20(3):265-269.

13. White JV, Guenter P, Jensen G, Malone A, Schofield M. Consensus statement: Academy of Nutrition and Dietetics and American Society for Parenteral and Enteral Nutrition: characteristics recommended for the identification and documentation of adult malnutrition (undernutrition). JPEN Journal of parenteral and enteral nutrition. 2012;36(3):275-283.

14. Allison SP, Lobo DN, Stanga Z. The treatment of hypoalbuminaemia. Clinical nutrition (Edinburgh, Scotland). 2001;20(3):275-279.

15. Kondrup J, Rasmussen HH, Hamberg O, Stanga Z. Nutritional risk screening (NRS 2002): a new method based on an analysis of controlled clinical trials. Clinical nutrition (Edinburgh, Scotland). 2003;22(3):321-336.

16. Cross MB, Yi PH, Thomas CF, Garcia J, Della Valle CJ. Evaluation of malnutrition in orthopaedic surgery. The Journal of the American Academy of Orthopaedic Surgeons. 2014;22(3):193-199.

17. Prenner G, Wasler A, Fahrleinter-Pammer A, et al. The role of serum albumin in the prediction of malnutrition in patients at least five yr after heart transplantation. Clinical transplantation. 2014;28(6):737-742.

18. Ikizler TA. Using and interpreting serum albumin and prealbumin as nutritional markers in patients on chronic dialysis. Seminars in dialysis. 2014;27(6):590-592. 
19. Schuetz $P$, Hausfater $P$, Amin D, et al. Optimizing triage and hospitalization in adult general medical emergency patients: the triage project. BMC emergency medicine. 2013;13:12.

20. Kondrup J, Allison SP, Elia M, Vellas B, Plauth M. ESPEN guidelines for nutrition screening 2002. Clinical nutrition (Edinburgh, Scotland). 2003;22(4):415-421.

21. Skipper A, Ferguson M, Thompson K, Castellanos VH, Porcari J. Nutrition screening tools: an analysis of the evidence. JPEN Journal of parenteral and enteral nutrition. 2012;36(3):292-298.

22. Bolayir B, Arik G, Yesil Y, et al. Validation of Nutritional Risk Screening-2002 in a Hospitalized Adult Population. Nutrition in clinical practice : official publication of the American Society for Parenteral and Enteral Nutrition. 2018.

23. Numeroso F, Barilli AL, Delsignore R. Prevalence and significance of hypoalbuminemia in an internal medicine department. European journal of internal medicine. 2008;19(8):587-591.

24. Gehring N, Imoberdorf R, Wegmann M, Ruhlin M, Ballmer PE. Serumalbumin--a qualified parameter to determine the nutritional status? Swiss Med Wkly. 2006;136(41-42):664-669.

25. Jensen GL, Mirtallo J, Compher C, et al. Adult starvation and disease-related malnutrition: a proposal for etiology-based diagnosis in the clinical practice setting from the International Consensus Guideline Committee. JPEN Journal of parenteral and enteral nutrition. 2010;34(2):156-159.

26. Jensen GL, Bistrian B, Roubenoff R, Heimburger DC. Malnutrition syndromes: a conundrum vs continuum. JPEN Journal of parenteral and enteral nutrition. 2009;33(6):710-716.

27. Jensen GL. Inflammation as the key interface of the medical and nutrition universes: a provocative examination of the future of clinical nutrition and medicine. JPEN Journal of parenteral and enteral nutrition. 2006;30(5):453-463.

28. Gabay C, Kushner I. Acute-phase proteins and other systemic responses to inflammation. The New England journal of medicine. 1999;340(6):448-454.

29. Library AoNaDEA. Academy of Nutrition and Dietetics Evidence Analysis Library. Nutrition Screening (NSCR): Serum Proteins. 2009;

http://www.andeal.org/topic.cfm?menu1/43584\&cat1/44302. Accessed March 22, 2018.

30. Budzynski J, Tojek K, Czerniak B, Banaszkiewicz Z. Scores of nutritional risk and parameters of nutritional status assessment as predictors of in-hospital mortality and readmissions in the general hospital population. Clinical nutrition (Edinburgh, Scotland). 2016;35(6):1464-1471.

31. Vincent JL, Dubois MJ, Navickis RJ, Wilkes MM. Hypoalbuminemia in acute illness: is there a rationale for intervention? A meta-analysis of cohort studies and controlled trials. Annals of surgery. 2003;237(3):319-334.

32. Kyle UG, Kossovsky MP, Karsegard VL, Pichard C. Comparison of tools for nutritional assessment and screening at hospital admission: a population study. Clinical nutrition (Edinburgh, Scotland). 2006;25(3):409-417.

33. Bauer JM, Vogl T, Wicklein S, Trogner J, Muhlberg W, Sieber CC. Comparison of the Mini Nutritional Assessment, Subjective Global Assessment, and Nutritional Risk Screening (NRS 2002) for nutritional screening and assessment in geriatric hospital patients. Zeitschrift fur Gerontologie und Geriatrie. 2005;38(5):322-327. 
Figures

Figure 1 Correlations of serum albumin and inflammation (log CRP) including graphical regression (panel A), serum albumin and nutritional risk (NRS 2002 total scores; panel B), and inflammation (log CRP) and nutritional risk (NRS 2002 total scores; panel C).

A.

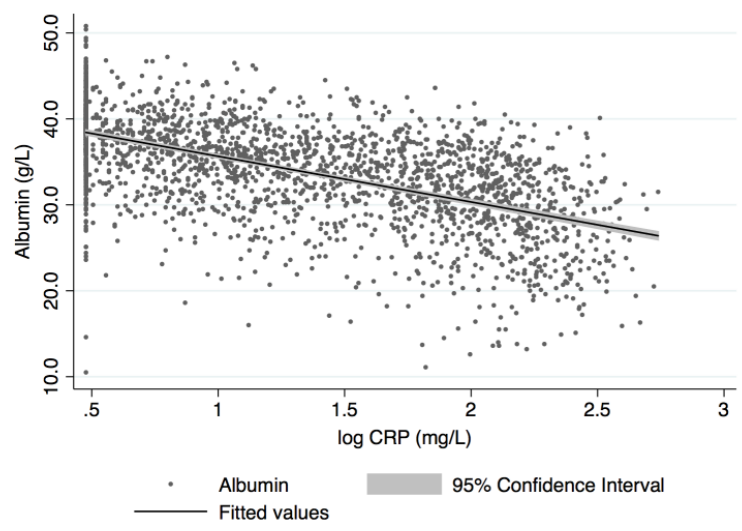

B.

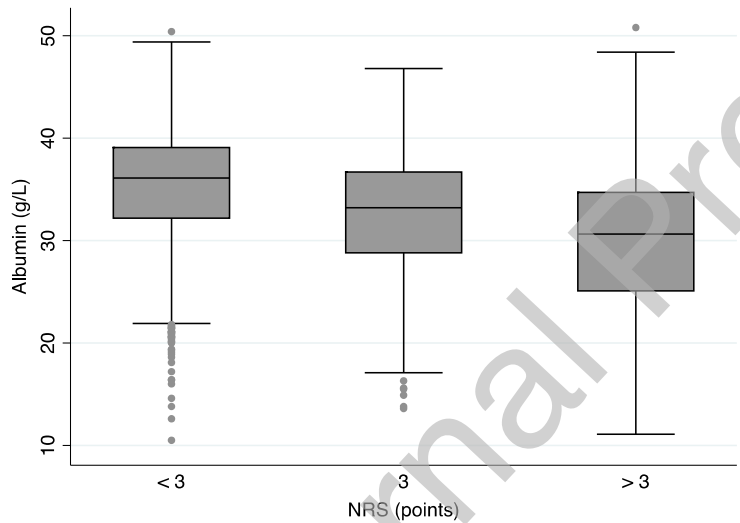

C.

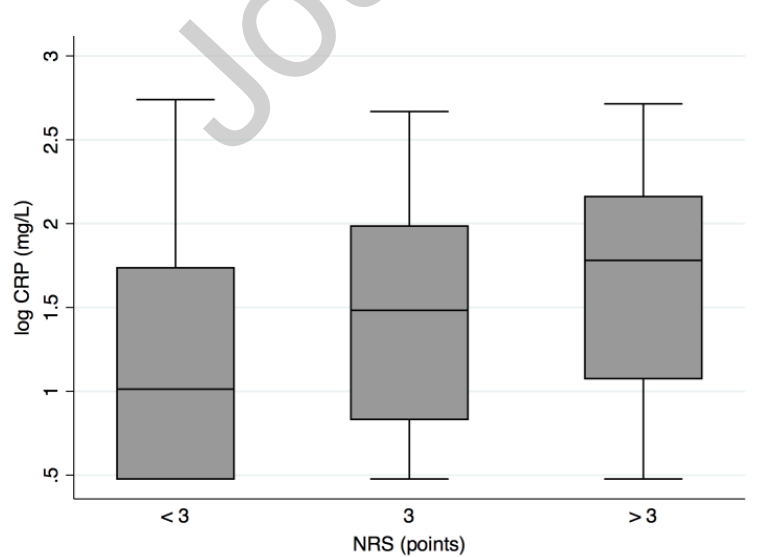

Figure 2 ROC statistics (AUC) of CRP values and NRS 2002 scores for the prediction of low serum albumin levels (panel A). ROC statistics (AUC) of CRP values, NRS 2002 scores, and serum albumin levels for the prediction of mortality (panel $B$ ).

A. 


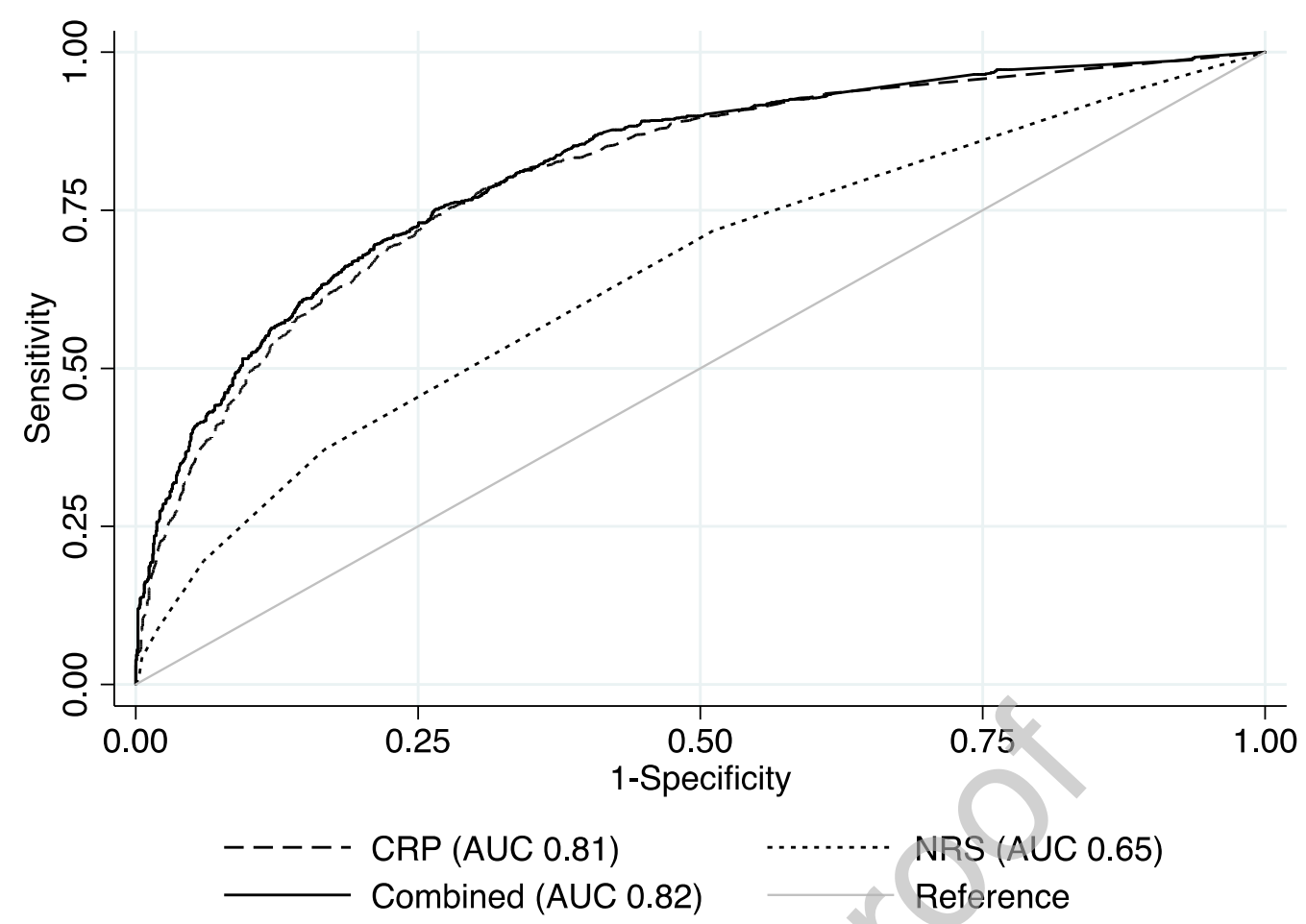

B.

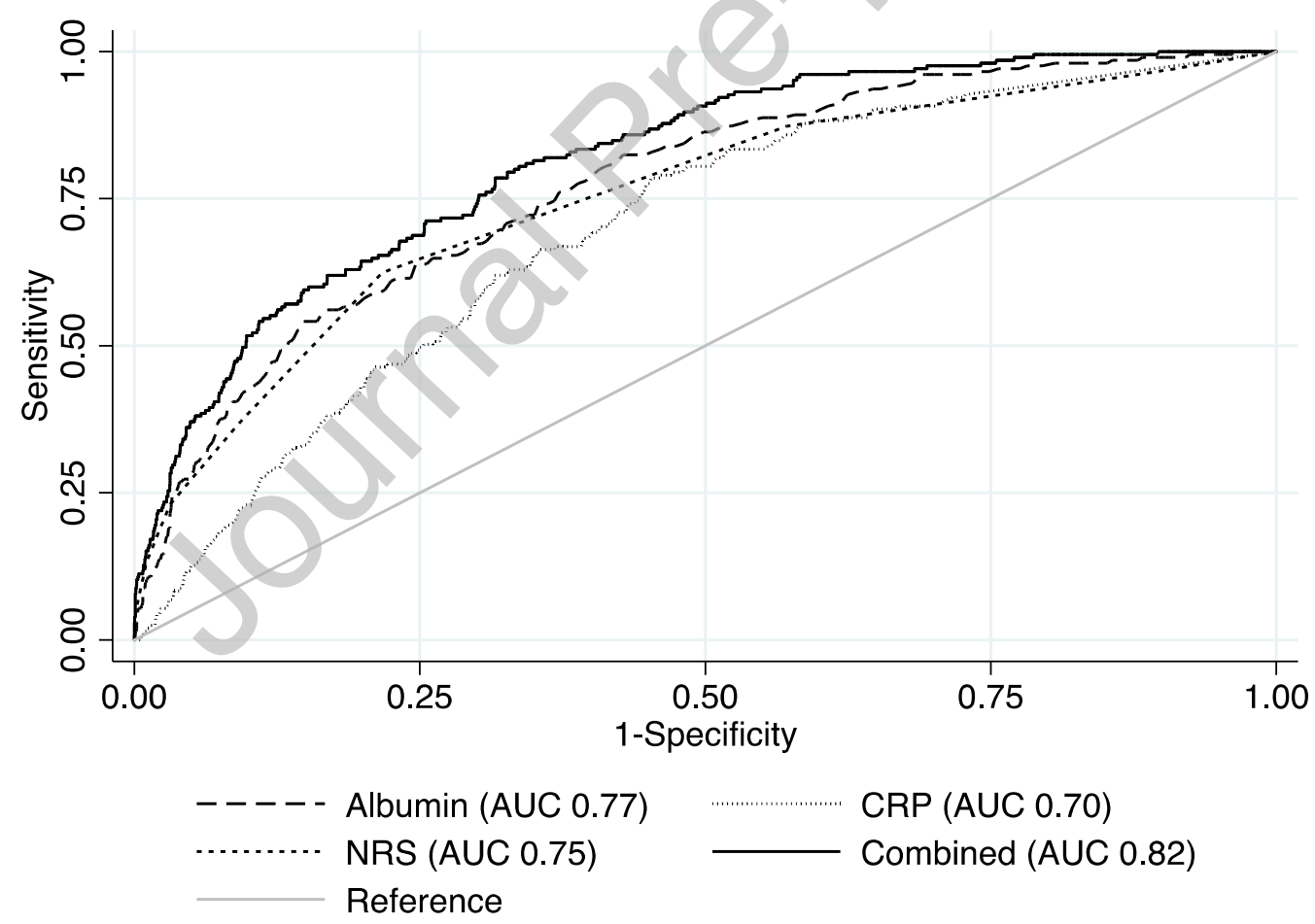

AUC, area under the curve; CRP, C-reactive protein; NRS, Nutritional Risk Screening 2002; ROC, receiver operating characteristic. 


\section{Tables}

Table 1 Baseline characteristics of the entire cohort, stratified by admission serum albumin levels, and stratified by vital status 30 days after admission

\begin{tabular}{|c|c|c|c|c|c|c|c|}
\hline & Total cohort & Serum albumin $\geq 34 \mathrm{~g} / \mathrm{L}$ & Serum albumin $<34 \mathrm{~g} / \mathrm{L}$ & P value & Survivors & Non-survivors & $P$ value \\
\hline Number of Patients, $n$ & 2465 & 1446 & 1019 & & 2260 & 205 & \\
\hline \multicolumn{8}{|l|}{ Socio-demographics } \\
\hline Female gender, $\mathrm{n}(\%)$ & $1076(43.7 \%)$ & $635(43.9 \%)$ & $441(43.3 \%)$ & 0.75 & $998(44.2 \%)$ & $78(38.0 \%)$ & 0.091 \\
\hline Age, median (IQR) & $71(60,80)$ & $70(56,80)$ & $72(63,80)$ & $<0.001$ & $71(59,79)$ & $76(68,84)$ & $<0.001$ \\
\hline \multicolumn{8}{|l|}{ Vital signs, median (IQR) } \\
\hline Blood pressure systolic $(\mathrm{mmHg})$ & $138.5(121,155)$ & $145(127,161)$ & $130(112,147)$ & $<0.001$ & $139(122,156)$ & $129(112,148)$ & $<0.001$ \\
\hline Blood pressure diastolic $(\mathrm{mmHg})$ & $80(69,90)$ & $83(73,94)$ & $74(65,86)$ & $<0.001$ & $80(70,91)$ & $74(65,87)$ & $<0.001$ \\
\hline Pulse rate $(\mathrm{bpm})$ & $85(72,100)$ & $82(69,95)$ & $89(77,105)$ & $<0.001$ & $84(71,99)$ & $90(77,109)$ & $<0.001$ \\
\hline $\mathrm{SpO} 2(\%)$ & $95(93,97)$ & $96(93,97)$ & $94(91,97)$ & $<0.001$ & $95(93,97)$ & $94(91,96)$ & $<0.001$ \\
\hline Temperature $\left({ }^{\circ} \mathrm{C}\right)$ & $37.0(36.6,37.6)$ & $36.9(36.5,37.3)$ & $37.2(36.7,38.0)$ & $<0.001$ & $37.0(36.6,37.6)$ & $37.1(36.6,37.7)$ & 0.74 \\
\hline Nutritional risk screening, $\mathrm{n}(\%)$ & & & & & & & \\
\hline NRS $2002=3$ & $333(13.5 \%)$ & $154(10.7 \%)$ & $179(17.6 \%)$ & & $281(12.4 \%)$ & $52(25.4 \%)$ & \\
\hline NRS $2002>3$ & $286(11.6 \%)$ & $87(6.0 \%)$ & $199(19.5 \%)$ & & $210(9.3 \%)$ & $76(37.1 \%)$ & \\
\hline \multicolumn{8}{|l|}{ Main diagnosis, $\mathrm{n}(\%)$} \\
\hline Cardiovascular disease & $684(27.7 \%)$ & $495(34.2 \%)$ & $189(18.5 \%)$ & $<0.001$ & $657(29.1 \%)$ & $27(13.2 \%)$ & $<0.001$ \\
\hline Digestive tract disease & $263(10.7 \%)$ & $134(9.3 \%)$ & $129(12.7 \%)$ & 0.007 & $248(11.0 \%)$ & $15(7.3 \%)$ & 0.10 \\
\hline Immune disorder & $49(2.0 \%)$ & $29(2.0 \%)$ & $20(2.0 \%)$ & 0.94 & $49(2.2 \%)$ & $0(0.0 \%)$ & 0.033 \\
\hline Infectious disease & $447(18.1 \%)$ & $193(13.3 \%)$ & $254(24.9 \%)$ & $<0.001$ & $414(18.3 \%)$ & $33(16.1 \%)$ & 0.43 \\
\hline Metabolic disorder & $63(2.6 \%)$ & $43(3.0 \%)$ & $20(2.0 \%)$ & 0.12 & $59(2.6 \%)$ & $4(2.0 \%)$ & 0.57 \\
\hline Miscellaneous & $88(3.6 \%)$ & $47(3.3 \%)$ & $41(4.0 \%)$ & 0.31 & $83(3.7 \%)$ & $5(2.4 \%)$ & 0.36 \\
\hline Musculoskeletal disorder & $103(4.2 \%)$ & $65(4.5 \%)$ & $38(3.7 \%)$ & 0.35 & $99(4.4 \%)$ & $4(2.0 \%)$ & 0.096 \\
\hline Neurologic disorder & $255(10.3 \%)$ & $214(14.8 \%)$ & $41(4.0 \%)$ & $<0.001$ & $236(10.4 \%)$ & $19(9.3 \%)$ & 0.60 \\
\hline Pulmonary disease & $133(5.4 \%)$ & $71(4.9 \%)$ & $62(6.1 \%)$ & 0.20 & $123(5.4 \%)$ & $10(4.9 \%)$ & 0.73 \\
\hline Tumor & $292(11.8 \%)$ & $90(6.2 \%)$ & $202(19.8 \%)$ & $<0.001$ & $206(9.1 \%)$ & $86(42.0 \%)$ & $<0.001$ \\
\hline Comorbidities, n (\%) & & & & & & & \\
\hline Chronic heart failure & $199(8.1 \%)$ & $97(6.7 \%)$ & $102(10.0 \%)$ & 0.003 & $177(7.8 \%)$ & $22(10.7 \%)$ & 0.14 \\
\hline Chronic renal failure & $445(18.1 \%)$ & $217(15.0 \%)$ & $228(22.4 \%)$ & $<0.001$ & $398(17.6 \%)$ & $47(22.9 \%)$ & 0.058 \\
\hline COPD & $147(6.0 \%)$ & $72(5.0 \%)$ & $75(7.4 \%)$ & 0.014 & $127(5.6 \%)$ & $20(9.8 \%)$ & 0.017 \\
\hline Coronary heart disease & $275(11.2 \%)$ & $208(14.4 \%)$ & $67(6.6 \%)$ & $<0.001$ & $259(11.5 \%)$ & $16(7.8 \%)$ & 0.110 \\
\hline Diabetes mellitus & $342(13.9 \%)$ & $198(13.7 \%)$ & $144(14.1 \%)$ & 0.76 & $321(14.2 \%)$ & $21(10.2 \%)$ & 0.12 \\
\hline Hypertension & $1248(50.6 \%)$ & $789(54.6 \%)$ & $459(45.0 \%)$ & $<0.001$ & $1168(51.7 \%)$ & $80(39.0 \%)$ & $<0.001$ \\
\hline Stroke & $183(7.4 \%)$ & $132(9.1 \%)$ & $51(5.0 \%)$ & $<0.001$ & $161(7.1 \%)$ & $22(10.7 \%)$ & 0.059 \\
\hline Tumor & $378(15.3 \%)$ & $144(10.0 \%)$ & $234(23.0 \%)$ & $<0.001$ & $307(13.6 \%)$ & $71(34.6 \%)$ & $<0.001$ \\
\hline \multicolumn{8}{|l|}{ Blood markers, median (IQR) } \\
\hline Serum albumin, $(\mathrm{g} / \mathrm{L})$ & $35.2(30.8,38.5)$ & $37.9(36.0,40.1)$ & $29.9(26.4,32.1)$ & $<0.001$ & $35.7(31.5,38.8)$ & $28.6(23.4,33.8)$ & $<0.001$ \\
\hline Hemoglobin, $(\mathrm{g} / \mathrm{L})$ & $131(111,145)$ & $138(126,150)$ & $115(99,131)$ & $<0.001$ & $132(113,145)$ & $115(99,132)$ & $<0.001$ \\
\hline
\end{tabular}




\begin{tabular}{|c|c|c|c|c|c|c|c|}
\hline Markers of inflammation & & & & & & & \\
\hline CRP continuous (mg/L) & $14(0.75)$ & $6(0,20)$ & $67(16,147)$ & $<0.001$ & $12(0,66)$ & $64(16,150)$ & $<0.001$ \\
\hline $\mathrm{CRP}<5 \mathrm{mg} / \mathrm{L}, \mathrm{n}(\%)$ & $790(32.1 \%)$ & $690(47.7 \%)$ & $100(9.8 \%)$ & $<0.001$ & $770(34.1 \%)$ & $20(9.8 \%)$ & $<0.001$ \\
\hline CRP $5.01-20$ mg/L, n (\%) & $589(23.9 \%)$ & $398(27.5 \%)$ & $191(18.7 \%)$ & & $549(24.3 \%)$ & $40(19.5 \%)$ & \\
\hline $\mathrm{CRP}>20 \mathrm{mg} / \mathrm{L}, \mathrm{n}(\%)$ & $1086(44.1 \%)$ & $358(24.8 \%)$ & $728(71.4 \%)$ & 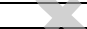 & $941(41.6 \%)$ & $145(70.7 \%)$ & \\
\hline PCT continuous (mg/L) & $.19(.11, .51)$ & $.14(.09, .24)$ & $.255(.13, .925)$ & $<0.001$ & $.18(.11, .485)$ & $.29(.15, .87)$ & $<0.001$ \\
\hline White blood cells $(\mathrm{G} / \mathrm{L})$ & $9.0(6.8,12.1)$ & $8.4(6.7,10.9)$ & $9.9(7.1,13.5)$ & $<0.001$ & $8.9(6.8,11.9)$ & $9.9(7.0,14.6)$ & 0.007 \\
\hline \multicolumn{8}{|l|}{ Kidney markers } \\
\hline Creatinine $(\mu \mathrm{mol} / \mathrm{L})$ & $95(76,126)$ & $92(75,114)$ & $101(78,150)$ & $<0.001$ & $94(76,124)$ & $107(74,163)$ & 0.007 \\
\hline Urea $(\mathrm{mmol} / \mathrm{L})$ & $6.9(5,10.5)$ & $6.5(4.8,9.1)$ & $7.8(5.3,13.2)$ & $<0.001$ & $6.8(5,10.1)$ & $9.6(5.9,14.9)$ & $<0.001$ \\
\hline
\end{tabular}

COPD, chronic obstructive pulmonary disease; CRP, C-reactive protein; IQR, interquartile range; NRS 2002, Nutritional Risk Screening 2002;

PCT, Procalcitonin.

Blood markers represent values at time of admission. 
Table 2 Regression analyses for associations of inflammation (CRP) and nutritional risk (NRS 2002) with hypoalbuminemia. Receiver operating characteristics $(A \cup C)$ for the prediction of hypoalbuminemia

\begin{tabular}{|c|c|c|c|c|c|c|c|c|}
\hline \multirow[b]{2}{*}{ Parameter } & \multicolumn{3}{|c|}{ Events, $\mathbf{n}(\%)$} & \multicolumn{4}{|c|}{ Regression analyses, OR $(95 \% \mathrm{Cl}), \boldsymbol{P}$ value } & \multirow[b]{2}{*}{ AUC $(95 \% \mathrm{Cl})$} \\
\hline & 30-day Mortality & $\begin{array}{c}\text { Serum albumin } \\
<34 \mathrm{~g} / \mathrm{L}\end{array}$ & $\underset{\substack{\text { Serum albumin } \\
\geq 34 \mathrm{~g} / \mathrm{L}}}{ }$ & Unadjusted & Model 1 & Model 2 & Model 3 & \\
\hline \multicolumn{9}{|l|}{$\mathrm{CRP}(\mathrm{mg} / \mathrm{L})$} \\
\hline$\leq 5$ & $20(2.5 \%)$ & $100(12.7 \%)$ & $690(87.3 \%)$ & Ref. & Ref. & Ref. & Ref. & \\
\hline $5.01-20.0$ & $40(6.8)$ & $191(32.4 \%)$ & $398(67.6 \%)$ & $3.31(2.52,4.34), P<0.001$ & $3.18(2.42,4.18), P<0.001$ & $2.69(1.88,3.83), P<0.001$ & $2.61(1.82,3.73), P<0.001$ & \\
\hline$>20$ & $145(13.4)$ & $728(67.0 \%)$ & $358(33.0 \%)$ & $14.03(10.98,17.93), P<0.001$ & $13.86(10.83,17.73), P<0.001$ & $10.51(7.51,14.72), P<0.001$ & $9.96(7.10,13.98), P<0.001$ & \\
\hline Continuous & & & & $6.59(5.65,7.70), P<0.001$ & $6.64(5.67,7.76), P<0.001$ & $6.03(4.84,7.52), P<0.001$ & $5.78(4.62,7.22), P<0.001$ & $0.81(0.79$ to 0.82$)$ \\
\hline \multicolumn{4}{|c|}{ NRS 2002 (points) } & & - & & & \\
\hline$<3$ & $77(4.2)$ & $641(34.7 \%)$ & $1205(65.3 \%)$ & Ref. & Ref. & Ref. & Ref. & \\
\hline 3 & $52(15.6)$ & $179(53.8 \%)$ & $154(46.2 \%)$ & $2.19(1.73,2.77), P<0.001$ & $2.01(1.58,2.55), P<0.001$ & $1.67(1.22,2.30), P=0,001$ & $1.51(1.07,2.13), P=0,018$ & \\
\hline$>3$ & $76(26.6)$ & $199(69.6 \%)$ & $87(30.4 \%)$ & $4.30(3.28,5.63), P<0.001$ & $4.07(3.10,5.33), P<0.001$ & $2.87(1.98,4.15), P<0.001$ & $2.40(1.62,3.54), P<0.001$ & \\
\hline Continuous & & & & $1.55(1.45,1.66), P<0.001$ & $1.52(1.41,1.63), P<0.001$ & $1.40(1.28,1.54), P<0.001$ & $1.33(1.19,1.47), P<0.001$ & 0.65 (0.62 to 0.67$)$ \\
\hline $\begin{array}{l}\text { Combined } \\
\text { (CRP \& NRS) } \\
\end{array}$ & & & & & & & & 0.82 (0.80 to 0.84$)$ \\
\hline
\end{tabular}

Adjustments: Model 1: age and sex; Model 2: age, sex, comorbidities, and main diagnosis; Model 3: fully adjusted for age, sex, comorbidities, main diagnosis, and CRP/NRS 2002.

For regression analysis with continuous values, CRP was log transformed with a base of ten before entering into statistical models. Therefore, the ORs correspond to a tenfold increase in CRP values.

95\% Cl, 95\% confidence interval; AUC, area under the curve; CRP, C-reactive protein; NRS 2002, Nutritional Risk Screening 2002; OR, odds ratio; Ref, reference. 
Table 3 Regression analyses for associations of serum albumin, inflammation (CRP), and nutritional risk (NRS 2002) with mortality, respectively. Receiver operating characteristics $(A \cup C)$ for the prediction of mortality 30 days after admission

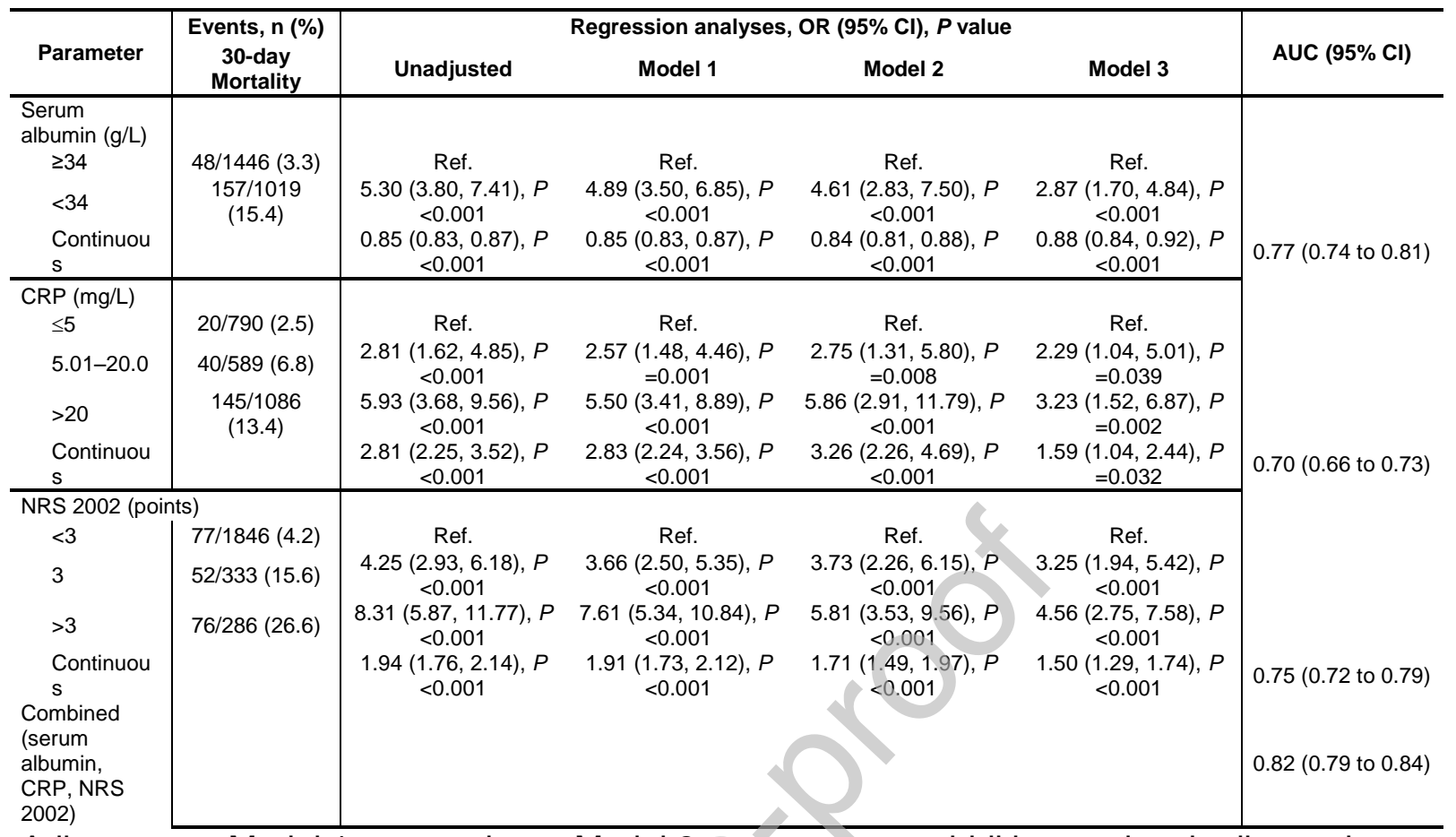

Adjustments: Model 1: age and sex; Model 2 age, sex, comorbidities, and main diagnosis;

Model 3: fully adjusted for age, sex, comorbidities, main diagnosis, and serum albumin/CRP/NRS 2002.

For regression analysis with continuous values, CRP was log transformed with a base of ten before entering into statistical models. Therefore, the ORs correspond to a tenfold increase in CRP values.

$95 \% \mathrm{Cl}, 95 \%$ confidence interval; AUC, area under the curve; CRP, C-reactive protein; NRS 2002, Nutritional Risk Screening 2002; OR, odds ratio; Ref, reference. 